\title{
Association between timing of kyphoplasty and opioid prescribing risk after vertebral fracture
}

\author{
Jay K. Nathan, MD, ${ }^{1}$ Mitchell A. Johnson, BS, ${ }^{2}$ Jennifer F. Waljee, MD, ${ }^{3}$ Nicholas Szerlip, MD, ${ }^{1}$ \\ Paul Park, MD,' and Mark E. Oppenlander, MD' \\ Departments of ${ }^{1}$ Neurosurgery and ${ }^{3}$ Surgery, Section of Plastic Surgery, University of Michigan, Ann Arbor, Michigan; and \\ 2Perelman School of Medicine, University of Pennsylvania, Philadelphia, Pennsylvania
}

\begin{abstract}
OBJECTIVE Approximately 550,000 Americans experience vertebral fracture annually, and most receive opioids to treat the resulting pain. Kyphoplasty of the fractured vertebra is a procedural alternative that may mitigate risks of even short-term opioid use. While reports of kyphoplasty's impact on pain scores are mixed, no large-scale data exist regarding opioid prescribing before and after the procedure. This study was conducted to determine whether timing of kyphoplasty following vertebral fracture is associated with duration or intensity of opioid prescribing.

METHODS This retrospective cohort study used 2001-2014 insurance claims data from a single, large private insurer in the US across multiple care settings. Patients were adults with vertebral fractures who were prescribed opioids and underwent balloon-assisted kyphoplasty within 4 months of fracture. Opioid overdose risk was stratified by prescribed average daily morphine milligram equivalents using CDC guidelines. Filled prescriptions and risk categories were evaluated at baseline and 90 days following kyphoplasty.

RESULTS Inclusion criteria were met by 7119 patients (median age 77 years, $71.7 \%$ female). Among included patients, 3505 (49.2\%) were opioid naïve before fracture. Of these patients, $31.1 \%$ had new persistent opioid prescribing beyond 90 days after kyphoplasty, and multivariable logistic regression identified kyphoplasty after 8 weeks as a predictor (OR $1.34,95 \% \mathrm{Cl} 1.02-1.76)$. For patients previously receiving opioids, kyphoplasty $>4$ weeks after fracture was associated with persistently elevated prescribing risk (OR 1.84, 95\% Cl 1.23-2.74).

CONCLUSIONS New persistent opioid prescribing occurred in nearly one-third of patients undergoing kyphoplasty after vertebral fracture, although early treatment was associated with a reduction in this risk. For patients not naïve to opioids before fracture diagnosis, early kyphoplasty was associated with less persistent elevation of opioid overdose risk. Subsequent trials must compare opioid use by vertebral fracture patients treated via operative (kyphoplasty) and nonoperative (ongoing opioid) strategies before concluding that kyphoplasty lacks value, and early referral for kyphoplasty may be appropriate to avoid missing a window of efficacy.
\end{abstract}

https://thejns.org/doi/abs/10.3171/2020.5.FOCUS20226

KEYWORDS drug addiction; elderly; kyphoplasty; opioids; pain relief; vertebral fracture

$\mathrm{V}$ ERTEBRAL fractures represent a significant burden of disease for older adults, affecting approximately 1.4 million people annually worldwide, and 550,000 people in the US. ${ }^{1,2}$ The most common clinical consequence of vertebral fractures is pain, in an estimated $84 \%$ of cases. ${ }^{3}$ The current initial standard of care after vertebral fracture is a combination of bed rest, analgesics, and external immobilization, 4,5 and opioids remain the most commonly used pharmacological analgesic. ${ }^{6}$

However, such treatments have been shown to leave $46 \%$ of vertebral fracture patients with insufficient pain relief. ${ }^{7}$ Moreover, even a short course of opioids may lead to persistent use, particularly in previously opioid-naïve patients. Recent research highlights persistent opioid prescribing as one of the most common risks of surgery for opioid-naïve patients, in whom only a short duration was intended. ${ }^{8}$ High-dose opioids carry not only immediate side effects but also long-term risks of dependence and overdose; these risks become heightened in already frail, elderly patients. ${ }^{9-11}$ For patients in significant pain after vertebral fracture, vertebral augmentation procedures such as kyphoplasty may provide relief.

ABBREVIATIONS CDC = US Centers for Disease Control and Prevention; $\mathrm{Cl}=$ confidence interval; $\mathrm{CPT}=$ Current Procedural Terminology; ICD = International Classification of Diseases; $M M E=$ morphine milligram equivalent; $\mathrm{OR}=$ odds ratio.

SUBMITTED March 20, 2020. ACCEPTED May 12, 2020.

INCLUDE WHEN CITING DOI: 10.3171/2020.5.FOCUS20226. 
Kyphoplasty is a highly standardized, minimally invasive procedure to expand a fractured and collapsed vertebral body with a balloon prior to the injection of cement via a needle. Data on kyphoplasty's efficacy for reducing pain have been conflicting, and may depend on early rather than delayed treatment after fracture, as indicated by randomized controlled trials. ${ }^{12}$ Improvement in pain should translate to decreased opioid requirements and cessation of prescribing, although this is not necessarily true and was not assessed directly in previous trials. If kyphoplasty is truly ineffective, it should have no benefit in reducing opioid prescribing, regardless of when it is performed. The focus of the present study is to determine whether opioid overdose risk from prescribing for vertebral fracture is indeed associated with timing of kyphoplasty.

\section{Methods \\ Data Set}

This study utilized the deidentified Clinformatics Data Mart database (OptumInsight), containing insurance claim information for inpatient and outpatient medical procedures and outpatient prescriptions for 56 million patients between 2001 and 2014 (inclusive). Medical service claims include the Current Procedural Terminology (CPT) code of the billed service, as well as the International Classification of Diseases (ICD) version-9 diagnosis codes for which the service was provided. This data set was chosen because it captures approximately $20 \%$ of the US privately insured population, including Medicare Advantage beneficiaries, across the continuum of inpatient and outpatient care. This permits creation of a timeline of both procedures and prescription medication. Use of this deidentified data set was deemed exempt from review by the University of Michigan IRB, and patient informed consent was not required.

\section{Study Criteria}

Adult patients were initially included if they had at least one medical service claim for CPT codes 22523 or 22524 , representing kyphoplasty performed at a thoracic or lumbar vertebra, respectively. Claims with CPT code 22525 represent treatment at an additional level, and total levels treated during a given procedure were tallied. Insurance coverage duration was queried, and only patients with 6 months of continuous coverage both before and after their first kyphoplasty were retained, to reduce the risk of incomplete data from short-term or discontinuous coverage. All medical service claims for these patients were queried to identify the date of first diagnosis of vertebral fracture based on ICD-9 code, and this was the date used to determine timing of interventions such as kyphoplasty and opioid prescribing. Only patients who underwent kyphoplasty within 4 months of fracture diagnosis were included, to ensure that at least 2 months of pre-kyphoplasty claims were available within the 6-month continuous coverage window. Any patient who had a claim for anesthesia for another operative procedure during the study period was excluded, to reduce confounding from opioid prescribing attributable to other operations or serious injuries. Claims for the dispensing of prescription medications were que- ried, and patients were included in the final analytical cohort only if they had filled at least one opioid prescription within 6 months of kyphoplasty, whether a standalone or combination drug, in tablet, solution, sublingual, or transdermal patch form. Figure 1 demonstrates inclusion and exclusion criteria along with patient numbers at each stage.

\section{Data Collection}

To examine duration and intensity of opioid prescribing in context, we categorized prescriptions into prefracture, postfracture, and postprocedure periods for each patient. The prefracture period provides the best assessment of baseline opioid prescribing. Patients lacking opioid prescriptions in the 2 months to 1 week before vertebral fracture were deemed opioid naïve, accounting for the possibility of receiving opioids for acute back pain days before the fracture was actually diagnosed. Opioid doses were converted to morphine milligram equivalents (MMEs) via published data ${ }^{13}$ If a prescription began in one period but carried into the subsequent period, MMEs were allocated proportionately to the respective periods assuming constant daily dosing. Intensity of prescribing within each period was calculated by averaging the total MME over the number of days any opioid was prescribed, accounting for potentially multiple concurrent prescriptions. Prescribing intensity was categorized into low, medium, high, and very high risk of opioid overdose-related death using exclusive ranges of $0-20,20-50,50-90$, and greater than 90 average daily MME, respectively, per US Centers for Disease Control and Prevention (CDC) guidelines for chronic pain management. ${ }^{14} \mathrm{CDC}$ categories are informative for capturing risk of death from overdose, with daily doses of 50 MMEs or higher associated with twice the risk as doses less than 20 MMEs. ${ }^{15}$ Clinicians are therefore advised to carefully reassess when prescribing 50 or more MMEs daily, and to avoid prescribing 90 or more MMEs daily unless there is a clear justification..$^{14}$ If a patient filled even one of these high-risk prescriptions in any period, prescribing was considered high-risk in that period regardless of average daily MME. A priori, opioid prescribing was defined as persistent if filled prescriptions continued beyond 90 days after kyphoplasty.

\section{Statistical Analysis}

Potential associations between persistent prescribing and individual demographic factors (age, sex, comorbid conditions, etc.) and treatment factors (interval from fracture to kyphoplasty) were investigated individually using the chi-square test, and those factors with a $p$ value $<0.05$ in the univariate association were included in multivariable logistic regression against the binary outcome of interest of returning to baseline opioid overdose risk or not. Subgroup analysis was performed for patients who were opioid naïve prior to vertebral fracture. Claim search and statistical analysis were performed using SAS software (version 9.4, SAS Institute, Inc.).

\section{Results}

A total of 7119 adult patients met inclusion criteria, with a median age of 77 years and similar distributions 


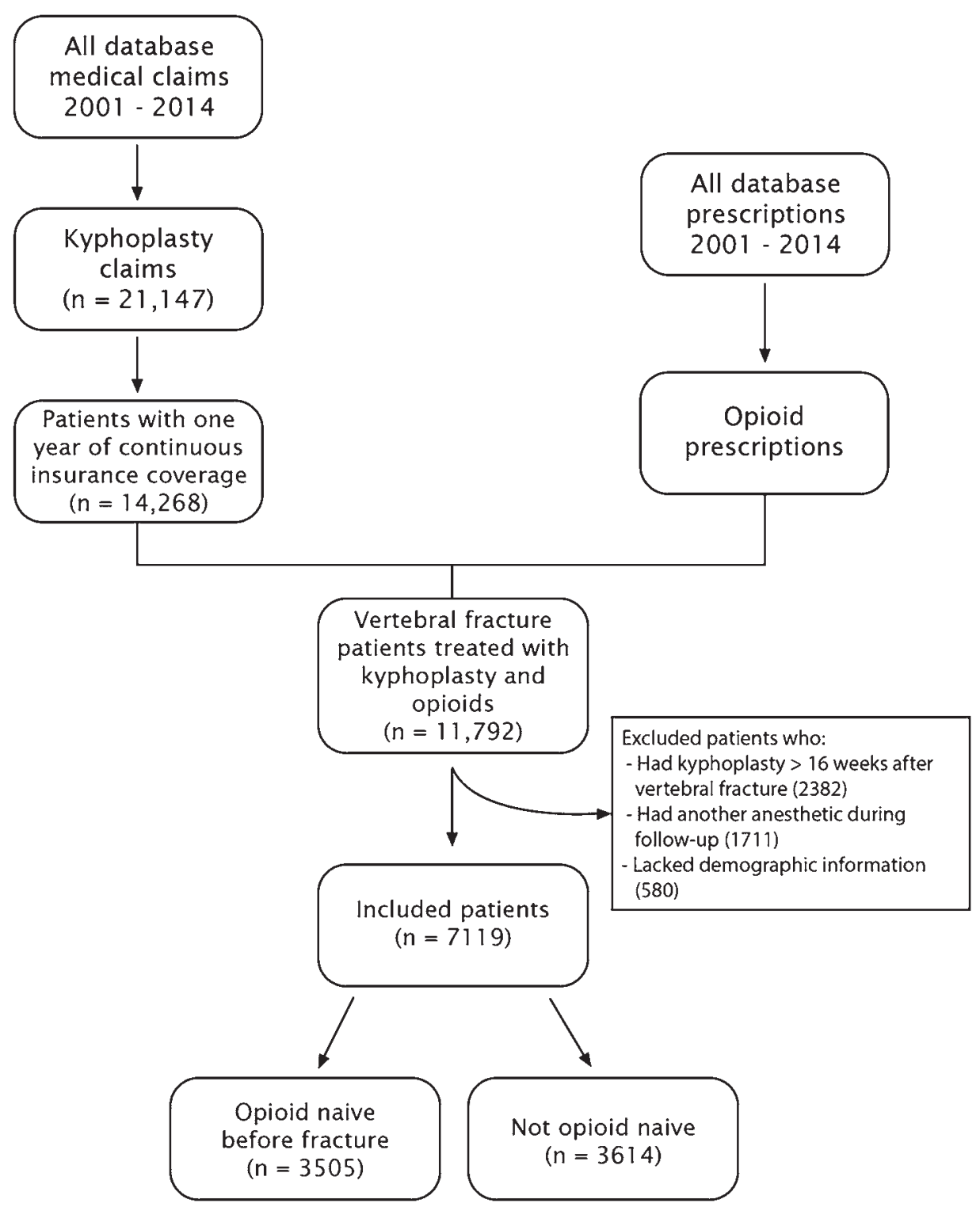

FIG. 1. Inclusion and exclusion criteria for identification of patients from insurance claims database.

of age, sex, race, insurance type, and opioid-naïve status, regardless of timing of kyphoplasty relative to vertebral fracture diagnosis (Table 1). Treatment groups did have statistically significant demographic differences in US census region and highest education level, with patients undergoing kyphoplasty within 4 weeks being more likely to have graduated college or higher. Of the 7119 patients, $3505(49.2 \%)$ were opioid naïve prior to fracture, and older patients were more likely to be opioid naïve $(\mathrm{p}<0.001)$. Kyphoplasty was used to treat a single vertebral level in $82 \%$ of cases overall, although multilevel kyphoplasty was more common in the later interval group. That group was also more likely to have a fracture in the thoracic versus lumbar region, although the majority of patients overall (53.4\%) underwent treatment for a lumbar spine fracture.

Figure 2 shows the opioid overdose risk category based on average daily prescribed MME for patients during the baseline (prefracture) period and after kyphoplasty. While 3505 patients were classified as opioid naïve, 899 were prescribed an opioid 7 days or less before vertebral fracture diagnosis, resulting in 2626 patients (36.9\%) with strictly no opioid exposure before documented diagnosis (Fig. 1). Only $13.1 \%$ had no opioid prescribed after kyphoplasty. The proportion of patients with opioid prescriptions at high risk or above increased by $73 \%$, from $10.5 \%$ at baseline to $18.2 \%$ after kyphoplasty. Nonetheless, among patients with high-risk opioid prescriptions after vertebral fracture, $55.7 \%$ had a reduction in their MME prescribing risk category following kyphoplasty (data not shown).

For patients who were previously opioid naïve, $31.1 \%$ had new persistent opioid prescribing beyond 90 days after kyphoplasty. Persistent opioid prescribing after kyphoplasty was more likely if it was performed 8-16 weeks after fracture diagnosis, compared to within 1 week (odds 
TABLE 1. Demographics and treatment characteristics of included patients

\begin{tabular}{|c|c|c|c|c|c|c|}
\hline \multirow[b]{2}{*}{ Variable } & \multirow[b]{2}{*}{ Overall } & \multicolumn{4}{|c|}{ Maximum Interval From Fracture Diagnosis to Kyphoplasty (wks) } & \multirow[b]{2}{*}{$\mathrm{p}$ Value } \\
\hline & & 1 & 4 & 8 & 16 & \\
\hline Age range, yrs & & & & & & 0.06 \\
\hline$<55$ & $460(6.5)$ & $175(6.6)$ & $152(5.9)$ & $71(6.2)$ & $62(7.9)$ & \\
\hline $55-64$ & $877(12.3)$ & $314(11.9)$ & $303(11.8)$ & $140(12.3)$ & $120(15.4)$ & \\
\hline $65-74$ & $1655(23.3)$ & $587(22.3)$ & $612(23.9)$ & $282(24.7)$ & $174(22.3)$ & \\
\hline$\geq 75$ & $4127(58)$ & $1558(59.2)$ & $1496(58.4)$ & $647(56.8)$ & $426(54.5)$ & \\
\hline Female & $5106(71.7)$ & $1920(72.9)$ & $1815(70.8)$ & $822(72.1)$ & $549(70.2)$ & 0.29 \\
\hline Race & & & & & & 0.10 \\
\hline White & $5820(81.8)$ & $2103(79.8)$ & $2125(82.9)$ & $933(81.8)$ & $659(84.3)$ & \\
\hline Black & 435 (6.1) & $174(6.6)$ & $148(5.8)$ & $66(5.8)$ & $47(6)$ & \\
\hline Hispanic & $485(6.8)$ & $201(7.6)$ & $157(6.1)$ & $77(6.8)$ & $50(6.4)$ & \\
\hline Asian & $133(1.9)$ & $49(1.9)$ & $52(2)$ & $22(1.9)$ & $10(1.3)$ & \\
\hline Unknown & $246(3.5)$ & $107(4.1)$ & $81(3.2)$ & $42(3.7)$ & $16(2.1)$ & \\
\hline US census region & & & & & & $<0.0001$ \\
\hline Midwest & 1319 (18.5) & $520(19.7)$ & $496(19.4)$ & $182(16)$ & $121(15.5)$ & \\
\hline Northeast & $418(5.9)$ & $124(4.7)$ & $151(5.9)$ & $87(7.6)$ & $56(7.2)$ & \\
\hline South & $3420(48)$ & $1329(50.5)$ & $1248(48.7)$ & $495(43.4)$ & $348(44.5)$ & \\
\hline West & $1784(25.1)$ & $600(22.8)$ & $610(23.8)$ & $340(29.8)$ & $234(29.9)$ & \\
\hline Unknown & $178(2.5)$ & $61(2.3)$ & $58(2.3)$ & $36(3.2)$ & $23(2.9)$ & \\
\hline Insurance type & & & & & & 0.13 \\
\hline Commercial & $2035(28.6)$ & $738(28)$ & $758(29.6)$ & $301(26.4)$ & $238(30.4)$ & \\
\hline Medicare Advantage & $5084(71.4)$ & $1896(72)$ & $1805(70.4)$ & $839(73.6)$ & $544(69.6)$ & \\
\hline Highest education level & & & & & & $<0.0001^{*}$ \\
\hline$<12$ th grade & $42(0.6)$ & $17(0.7)$ & $15(0.6)$ & $6(0.5)$ & $4(0.5)$ & \\
\hline High school diploma & $2315(32.5)$ & $882(33.5)$ & $809(31.6)$ & $368(32.3)$ & $256(32.7)$ & \\
\hline Some college & $3905(54.9)$ & $1403(53.3)$ & $1424(55.6)$ & $641(56.2)$ & $437(55.9)$ & \\
\hline Bachelor's degree+ & $846(11.9)$ & $327(12.4)$ & $311(12.1)$ & $123(10.8)$ & $85(10.9)$ & \\
\hline Unknown & $11(0.15)$ & $5(0.2)$ & $4(0.2)$ & $2(0.2)$ & 0 & \\
\hline Opioid naïve & $3505(49.2)$ & $1249(47.4)$ & $1284(50.1)$ & $566(49.7)$ & $406(51.9)$ & 0.09 \\
\hline High-risk opioid exposure before fracture & $1394(19.6)$ & $577(21.9)$ & $464(18.1)$ & $204(17.9)$ & $149(19.1)$ & 0.002 \\
\hline Spinal region treated & & & & & & $<0.0001$ \\
\hline Thoracic & $3315(46.6)$ & $1081(41)$ & $1251(48.8)$ & $583(51.1)$ & $400(51.2)$ & \\
\hline Lumbar & $3804(53.4)$ & $1553(59)$ & $1312(51.2)$ & $557(48.9)$ & $382(48.9)$ & \\
\hline Multilevel kyphoplasty & $1281(18)$ & $398(15.1)$ & $460(18)$ & $222(19.5)$ & $201(25.7)$ & $<0.0001$ \\
\hline Total & 7119 & $2634(37)$ & $2563(36)$ & $1140(16)$ & $782(11)$ & \\
\hline
\end{tabular}

Data given as value (\%) unless otherwise indicated.

* Fisher's exact test.

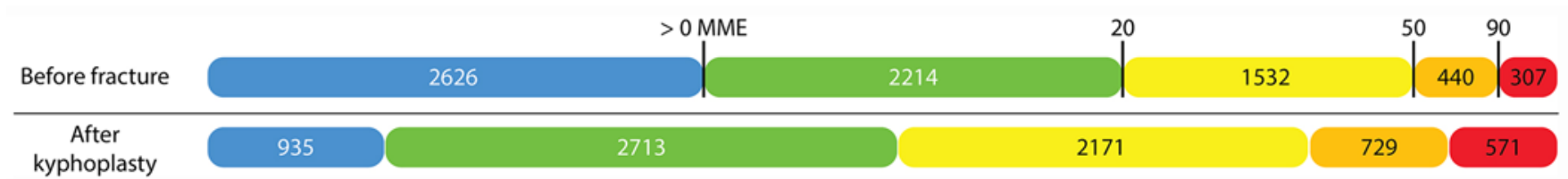

FIG. 2. CDC categories of opioid overdose risk before date of fracture diagnosis and up to 6 months after kyphoplasty. Blue represents no opioids prescribed, green low-risk prescribing, yellow medium-risk, orange high-risk, and red very high-risk. Patient numbers are shown within each segment, sized to scale. Of note, patients who received their first opioid prescription in the 7 days before fracture diagnosis are not included in the top blue (no-opioid) segment but were classified as opioid naïve, because they lacked chronic exposure and may have been treated for new back pain prior to documented vertebral fracture. 
TABLE 2. Multivariable logistic regression predicting filling of any opioid prescription by opioid-naïve patients more than 90 days after kyphoplasty

\begin{tabular}{|c|c|c|c|}
\hline Characteristic & OR $(95 \% \mathrm{Cl})$ & SE & $p$ Value \\
\hline \multicolumn{4}{|l|}{ Kyphoplasty performed within } \\
\hline $1 \mathrm{wk}$ & 1 & NA & NA \\
\hline 4 wks & $0.86(0.70-1.04)$ & 0.10 & 0.12 \\
\hline 8 wks & $1.20(0.94-1.53)$ & 0.12 & 0.14 \\
\hline 16 wks & $1.34(1.02-1.76)$ & 0.14 & 0.03 \\
\hline \multicolumn{4}{|l|}{ Age range, yrs } \\
\hline$<55$ & 1 & NA & NA \\
\hline 55 to 64 & $0.70(0.49-1.02)$ & 0.19 & 0.06 \\
\hline 65 to 74 & $0.80(0.57-1.12)$ & 0.17 & 0.20 \\
\hline$\geq 75$ & $0.77(0.56-1.05)$ & 0.16 & 0.10 \\
\hline Female & $1.44(1.21-1.72)$ & 0.09 & $<0.0001$ \\
\hline \multicolumn{4}{|l|}{ Pain diagnoses } \\
\hline Neck & $1.33(1.08-1.62)$ & 0.10 & $<0.01$ \\
\hline Other & $1.07(0.92-1.25)$ & 0.08 & 0.40 \\
\hline Substance abuse diagnosis & $1.46(0.98-2.17)$ & 0.20 & 0.06 \\
\hline Tobacco use diagnosis & $1.04(0.83-1.30)$ & 0.11 & 0.73 \\
\hline Psychiatric diagnosis & $1.24(1.04-1.47)$ & 0.09 & 0.01 \\
\hline Charlson Comorbidity Index & $1.07(1.04-1.10)$ & 0.02 & $<0.0001$ \\
\hline \multicolumn{4}{|l|}{ Spinal region treated } \\
\hline Thoracic & 1 & NA & NA \\
\hline Lumbar & $0.94(0.81-1.1)$ & 0.08 & 0.46 \\
\hline \multicolumn{4}{|c|}{$\begin{array}{l}\text { Average daily MME opioid dose } \\
\text { (after fracture, before kyphoplasty) }\end{array}$} \\
\hline 0 & 1 & NA & NA \\
\hline$<20$ & $1.19(0.95-1.49)$ & 0.12 & 0.13 \\
\hline $20-50$ & $1.34(1.08-1.67)$ & 0.11 & 0.01 \\
\hline $50-90$ & $1.32(0.97-1.79)$ & 0.16 & 0.08 \\
\hline$\geq 90$ & $1.77(1.23-2.54)$ & 0.19 & $<0.01$ \\
\hline $\begin{array}{l}\text { Any high-risk opioid exposure } \\
\text { after kyphoplasty }\end{array}$ & $2.72(2.32-3.19)$ & 0.08 & $<0.0001$ \\
\hline
\end{tabular}

$\mathrm{NA}=$ not applicable; $\mathrm{SE}=$ standard error.

ratio [OR] 1.34, 95\% confidence interval [CI] 1.02-1.76; Table 2). It was also more likely if high-risk opioid prescribing occurred before (OR 1.77, 95\% CI 1.23-2.54) or after (OR 2.72, 95\% CI 2.32-3.19) kyphoplasty. Nonmodifiable factors also associated with persistent prescribing for the opioid-naïve patient include female sex (OR 1.44, 95\% CI 1.21-1.72), previous neck pain (OR 1.33, 95\% CI $1.08-1.62$ ) or psychiatric (OR $1.24,95 \%$ CI 1.04-1.47) diagnoses, and higher Charlson Comorbidity Index (OR $1.07,95 \%$ CI 1.04-1.10).

For patients who were not opioid naïve prior to their vertebral fracture, Table 3 models the odds of persistent opioid prescribing at a risk category above the patient's baseline. Compared to patients who underwent kyphoplasty within 1 week of fracture, patients who had kyphoplasty 4-8 weeks (OR 1.84, 95\% CI 1.23-2.74) or 8-16 weeks (OR 1.84, 95\% CI 1.16-2.92) after fracture were more likely to remain at higher risk of overdose than their
TABLE 3. Multivariable logistic regression predicting opioid prescribing risk higher than baseline 90 days after kyphoplasty

\begin{tabular}{|c|c|c|c|}
\hline Characteristic & OR $(95 \% \mathrm{Cl})$ & SE & $p$ Value \\
\hline \multicolumn{4}{|l|}{ Kyphoplasty performed within } \\
\hline 1 wk & 1 & NA & NA \\
\hline 4 wks & $1.18(0.87-1.61)$ & 0.16 & 0.29 \\
\hline 8 wks & $1.84(1.23-2.74)$ & 0.20 & 0.003 \\
\hline 16 wks & $1.84(1.16-2.92)$ & 0.24 & 0.01 \\
\hline \multicolumn{4}{|l|}{ Age range, yrs } \\
\hline$<55$ & 1 & NA & NA \\
\hline $55-64$ & $1.08(0.63-1.84)$ & 0.27 & 0.78 \\
\hline $65-74$ & $0.73(0.44-1.19)$ & 0.25 & 0.21 \\
\hline$\geq 75$ & $0.62(0.39-1.00)$ & 0.24 & 0.05 \\
\hline Female & $0.80(0.60-1.08)$ & 0.15 & 0.15 \\
\hline \multicolumn{4}{|l|}{ Pain diagnoses } \\
\hline Neck & $1.08(0.78-1.48)$ & 0.16 & 0.64 \\
\hline Other & $1.32(1.01-1.72)$ & 0.14 & 0.04 \\
\hline Substance abuse diagnosis & $1.32(0.81-2.14)$ & 0.25 & 0.27 \\
\hline Tobacco use diagnosis & $0.87(0.62-1.24)$ & 0.18 & 0.44 \\
\hline Psychiatric diagnosis & $1.31(0.99-1.73)$ & 0.14 & 0.06 \\
\hline Charlson Comorbidity Index & $1.03(0.98-1.07)$ & 0.02 & 0.27 \\
\hline \multicolumn{4}{|l|}{ Spinal region treated } \\
\hline Thoracic & 1 & NA & NA \\
\hline Lumbar & $0.90(0.69-1.18)$ & 0.13 & 0.45 \\
\hline $\begin{array}{l}\text { Any high-risk opioid exposure after } \\
\text { fracture, before kyphoplasty }\end{array}$ & $0.71(0.52-0.97)$ & 0.16 & 0.03 \\
\hline
\end{tabular}

baseline. Preexisting pain diagnoses other than neck pain were also associated with persistently elevated opioid risk (OR 1.32, 95\% CI 1.01-1.72), while any high-risk exposure to opioids in the period between fracture and kyphoplasty was associated with less risk (OR $0.71,95 \%$ CI 0.52-0.97).

\section{Discussion}

Kyphoplasty is used to treat pain following vertebral fracture, and in this study we found that a majority of patients who received high-risk opioid prescriptions after vertebral fracture had a reduction in prescribing risk after kyphoplasty. When opioid-naïve patients underwent kyphoplasty between 8 and 16 weeks after fracture, there was an associated 34\% increase in risk of new persistent opioid prescribing compared to those with earlier intervention. When patients who were previously prescribed opioids had the procedure 4 or more weeks after fracture, there was an associated $84 \%$ increase in riskier opioid prescribing by 90 days. Taken together, these results suggest that especially for vertebral fracture patients at high risk of opioid overdose or serious side effects, early rather than delayed kyphoplasty may be more beneficial in reducing opioid exposure.

We defined opioid prescribing beyond 90 days after kyphoplasty as persistent because we expect analgesic effects of kyphoplasty (if any) to have occurred by that point, and we do not anticipate significant ongoing pain 
related to the procedure itself. In our study, risk factors for persistent opioid filled prescriptions among previously opioid-naïve patients include female sex, history of neck pain or psychiatric disorder, higher Charlson Comorbidity Index, higher opioid dose after fracture, and, importantly, longer interval from fracture to kyphoplasty. This finding persists even after controlling for high-dose opioid prescribing after kyphoplasty, which is an independent risk factor for prolonged prescribing.

Prior randomized controlled trials have demonstrated pain relief from kyphoplasty after vertebral fracture when performed in the acute setting. ${ }^{16}$ However, with greater heterogeneity in treatment time, up to 1 year, other studies have failed to show a significant effect, suggesting more consistent efficacy with early rather than delayed procedures. ${ }^{17,18}$ None of these trials reported opioid use or prescribing as outcomes, which is essential when comparing the clinical utility of an invasive procedure versus ongoing medical management to treat pain. In this study we found that nearly one-third of patients who were opioid naïve before their vertebral fracture had opioids prescribed more than 90 days following kyphoplasty, an alarmingly high proportion. This is particularly noteworthy in an era of heightened caution around opioids, new awareness of perioperative opioid use becoming persistent, and new regulations restricting opioid prescribing.

Opioid prescription intensity and duration serve as surrogate measures for pain after vertebral fracture, but measuring prescribing levels directly adds value beyond prior studies by capturing ongoing use that may not be pain-related. Persistent and high-dose opioid use directly increase patients' risks of side effects and death from overdose, regardless of the indication for prescribing. Variability in pain response to spine interventions based on intensity of preoperative opioid use has been documented previously in the setting of lumbar spine procedures for degenerative disease. ${ }^{19}$ Thus, subgrouping patients based on opioid prescribing prior to fracture, and controlling for prescribing levels after fracture but before kyphoplasty, was critical to examining the association of time to kyphoplasty independently.

This retrospective study of insurance claims data is not without limitations. First, patients aged 65 years and older in the US are likely to qualify for government-sponsored health insurance via Medicare and would only be included in this private insurance database if they opted for a Medicare Advantage or other commercial health plan from this particular carrier. While this may affect generalizability to all elderly patients, Medicare beneficiaries across socioeconomic strata have access to Medicare Advantage plans, and the large number of included patients reduces bias. Next, studying opioid consumption directly would be ideal, but it is not possible from these data to know what proportion of prescribed medication was ultimately taken by each patient. We focus instead on classifying risks of exposure to opioids via filled prescriptions; prescriptions that may have been written but not filled are not included in these analyses. Furthermore, assuming no widespread illegal activity such as diversion or black market purchases, the absence of an opioid prescription does signal the absence of opioid consumption. Given the large proportion of prescribed but unused opioids among the US population, ${ }^{20,21}$ reductions in opioid prescribing are also valuable per se from a public health standpoint..$^{22,23}$

Future work must examine actual opioid use in this population, clarifying exact doses and risks to individual patients, stratified by fracture severity. This should occur together with postoperative pain-level assessments and monitoring for opioid use disproportionate to pain, a signal of opioid misuse.

\section{Conclusions}

The public health burden of vertebral fractures is large and will continue to grow in an aging population. Pain is a common symptom of vertebral fracture, very often requiring opioid analgesia. Yet the burden of opioid use and overdose in the US is of grave concern, mandating alternative therapeutic options. Kyphoplasty is one nonopioid tool to address painful vertebral fracture, particularly in the acute setting. In this insurance claims-based analysis, we found that patients who underwent kyphoplasty early rather than after a prolonged course of nonoperative management had less risk of persistently elevated opioid prescribing more than 90 days after the procedure. While many have deemed kyphoplasty ineffective, prior trials did not consider the risks of the alternative, opioid-based treatment strategies and may have missed a potentially key metric of the procedure's value.

\section{Acknowledgments}

We thank Dr. Megan Foldenauer for assistance with illustrations, and May $\mathrm{Hu}$ for her statistical expertise and sharing of code used for portions of the database query.

\section{References}

1. Johnell O, Kanis JA. An estimate of the worldwide prevalence and disability associated with osteoporotic fractures. Osteoporos Int. 2006;17(12):1726-1733.

2. Burge R, Dawson-Hughes B, Solomon DH, et al. Incidence and economic burden of osteoporosis-related fractures in the United States, 2005-2025. J Bone Miner Res. 2007;22(3): 465-475.

3. Silverman SL. The clinical consequences of vertebral compression fracture. Bone. 1992;13(suppl 2):S27-S31.

4. Goldstein CL, Chutkan NB, Choma TJ, Orr RD. Management of the elderly with vertebral compression fractures. Neurosurgery. 2015;77(suppl 4):S33-S45.

5. Klazen CA, Verhaar HJ, Lohle PN, et al. Clinical course of pain in acute osteoporotic vertebral compression fractures. $J$ Vasc Interv Radiol. 2010;21(9):1405-1409.

6. Megale RZ, Pollack A, Britt H, et al. Management of vertebral compression fracture in general practice: $\mathrm{BEACH}$ program. PLoS One. 2017;12(5): 0176351.

7. Venmans A, Lohle PN, van Rooij WJ. Pain course in conservatively treated patients with back pain and a VCF on the spine radiograph (VERTOS III). Skeletal Radiol. 2014;43(1): $13-18$.

8. Brummett CM, Waljee JF, Goesling J, et al. New persistent opioid use after minor and major surgical procedures in US adults. JAMA Surg. 2017;152(6):e170504.

9. Daoust R, Paquet J, Moore L, et al. Incidence and risk factors of long-term opioid use in elderly trauma patients. Ann Surg. 2018;268(6):985-991.

10. Shorr RI, Griffin MR, Daugherty JR, Ray WA. Opioid anal- 
gesics and the risk of hip fracture in the elderly: codeine and propoxyphene. J Gerontol. 1992;47(4):M111-M115.

11. Seppala LJ, van de Glind EMM, Daams JG, et al. Fall-riskincreasing drugs: a systematic review and meta-analysis: III. Others. J Am Med Dir Assoc. 2018;19(4):372.e1-372.e8.

12. Chandra RV, Maingard J, Asadi H, et al. Vertebroplasty and kyphoplasty for osteoporotic vertebral fractures: What are the latest data? AJNR Am J Neuroradiol. 2018;39(5):798-806.

13. Vieweg WV, Lipps WF, Fernandez A. Opioids and methadone equivalents for clinicians. Prim Care Companion J Clin Psychiatry. 2005;7(3):86-88.

14. Dowell D, Haegerich TM, Chou R. CDC Guideline for Prescribing Opioids for Chronic Pain-United States, 2016. JAMA. 2016;315(15):1624-1645.

15. Calculating Total Daily Dose of Opioids for Safer Dosage. US Centers for Disease Control and Prevention. Accessed June 22, 2020. www.cdc.gov/drugoverdose/prescribing/guideline.html

16. Clark W, Bird P, Gonski P, et al. Safety and efficacy of vertebroplasty for acute painful osteoporotic fractures (VAPOUR): a multicentre, randomised, double-blind, placebocontrolled trial. Lancet. 2016;388(10052):1408-1416.

17. Buchbinder R, Osborne RH, Ebeling PR, et al. A randomized trial of vertebroplasty for painful osteoporotic vertebral fractures. N Engl J Med. 2009;361(6):557-568.

18. Kallmes DF, Comstock BA, Heagerty PJ, et al. A randomized trial of vertebroplasty for osteoporotic spinal fractures. $N$ Engl J Med. 2009;361(6):569-579.

19. Wick JB, Sivaganesan A, Chotai S, et al. Is there a preoperative morphine equianalgesic dose that predicts ability to achieve a clinically meaningful improvement following spine surgery? Neurosurgery. 2018;83(2):245-251.

20. Feinberg AE, Chesney TR, Srikandarajah S, et al. Opioid use after discharge in postoperative patients: A systematic review. Ann Surg. 2018;267(6):1056-1062.

21. Bicket MC, Long JJ, Pronovost PJ, et al. Prescription opioid analgesics commonly unused after surgery: a systematic review. JAMA Surg. 2017;152(11):1066-1071.

22. Han B, Compton WM, Blanco C, et al. Prescription opioid use, misuse, and use disorders in U.S. adults: 2015 national survey on drug use and health. Ann Intern Med. 2017;167(5): 293-301.
23. Egan KL, Gregory E, Sparks M, Wolfson M. From dispensed to disposed: evaluating the effectiveness of disposal programs through a comparison with prescription drug monitoring program data. Am J Drug Alcohol Abuse. 2017;43(1): $69-77$.

\section{Disclosures}

Dr. Oppenlander reports consultation for Globus Medical, DePuy Spine, and LifeNet Health. Dr. Park reports consultation for Globus Medical and NuVasive, royalties received from Globus Medical for an unrelated device, and support of non-study-related clinical or research effort from DePuy and ISSG.

\section{Author Contributions}

Conception and design: Oppenlander, Nathan, Waljee, Park. Acquisition of data: Oppenlander, Nathan, Waljee. Analysis and interpretation of data: Oppenlander, Nathan, Waljee. Drafting the article: Oppenlander, Nathan, Johnson. Critically revising the article: Oppenlander, Waljee, Szerlip, Park. Reviewed submitted version of manuscript: all authors. Approved the final version of the manuscript on behalf of all authors: Oppenlander. Statistical analysis: Oppenlander, Nathan, Waljee. Study supervision: Oppenlander.

\section{Supplemental Information}

Previous Presentations

Preliminary work on this study was presented at the Spine Summit, March 14-17, 2019, in Miami, Florida.

\section{Correspondence}

Mark E. Oppenlander: University of Michigan, Ann Arbor, MI. moppenla@med.umich.edu. 